\title{
A model for analysing the dynamics of the second wave of corona virus (COVID-19) in Nigeria
}

\author{
Ashiribo Senapon WUSU ${ }^{a, *}$, Olusola Aanu OLABANJOb ${ }^{\mathrm{b}}$, Moses Adebowale AKANBI ${ }^{\mathrm{a}}$ \\ ${ }^{a}$ Department of Mathematics, Lagos State University, Lagos, Nigeria. \\ ${ }^{b}$ Department of Mathematics, Morgan State University, Maryland, USA.
}

\begin{abstract}
The resurgence of the coronavirus (COVID-19) disease is rapidly taking its toll on many nations across the globe. As the situation rapidly evolves, the entire world is faced with difficult and challenging moment and the need to be safe from the disease. Several international efforts are geared towards the production of effective vaccine for curing the disease. But, pending the availability of such vaccine, the need to flatten the curves is now the top priority of both governmental and non-governmental organisations in Nigeria. Estimation of the basic reproduction number of the disease is crucial to understanding its dynamics and making suitable preventive policies that will slow the spread and ultimately flatten the curves. In this work, we propose a compartmental-based model for analysing the dynamics of the pandemics' second wave in Nigeria taking into consideration, the current control measures. Using the Quasi-Newton algorithm, the model is fitted to the available data provided by Nigeria Centre for Disease Control (NCDC), World Health Organization (WHO) and accessible on the Wolfram Data Repository. The basic reproduction number of the second wave of the disease in Nigeria is estimated using the model. The model was also used to estimate the infection rate, average latent time, average recovery rate and average mortality rate of the disease. Efficiency of the current control measures is also measured. Forecast of the turning points and possible vanishing time of the virus in Nigeria are made. The infection rate, average latent time, average recovery rate and average mortality rate of the disease are estimated. Also, the basic reproduction number of the disease in Nigeria is estimated. Predictions on the turning points and possible vanishing time of the virus in Nigeria are made. Recommendations on how to manage the resurgence of the disease in Nigeria are also suggested.
\end{abstract}

Keywords: Coronavirus, COVID-19, pandemic, second Wave, Nigeria.

2020 MSC: 65L05, 37M05, 49M37.

(C)2022 All rights reserved.

\section{Introduction}

The resurgence of the novel CoronaVirus (COVID-19) pandemic in Nigeria and the world at large has raised the quest for standard and scientific methods in the provision of suitable solution to a pandemic is of great concern especially. In understanding the dynamics of an epidemic, $[7,10]$ showed that epidemiological models are particularly suitable. Compartmental-based models have been shown to be effective in epidemiological modeling $[3,13]$ for predicting, determining, validating and analyzing the

\footnotetext{
*Corresponding author

Email addresses: ashiribo. wusu@lasu.edu.ng (Ashiribo Senapon WUSU), olola57@morgan.edu (Olusola Aanu OLABANJO), moses.akanbi@lasu.edu.ng (Moses Adebowale AKANBI)
}

doi: $10.22436 /$ jmcs.026.01.03

Received: 2021-02-14 Revised: 2021-04-16 Accepted: 2021-08-10 
rate of susceptibility, exposure, infection, recovery and mortality of a pandemic [7]. Usually, it is assumed that the incidence rate of a disease is given by an unspecified nonlinear function constrained by a few biologically motivated conditions. Due to the uniqueness of the Coronavirus pandemic, assessing the impacts of responses and determining a number of qualitative parameters of the disease has made this work a bit complex. Like other nations, the resurgence of the pandemic in Nigeria has been met with renewed enforcement of some social and health policies [1]. These measures are steps to reducing the contact parameter of the disease $[8,21]$. With the daily cases on the rise, it becomes necessary to understand the dynamics of the pandemic and hence, provide the best strategy to slow the spread and eventually flatten the curves in Nigeria. Ordinary differential equations which are deterministic in nature $[11,14,16]$ are often use in epidemic models to analyse the dynamics of a disease by estimating the crucial parameters of the model [18]. Authors in [2,4] have presented models to visualize the geographical spread of the disease. Many authors have proposed several epidemiological models [5, 9, 15, 19, 20, 22] in the efforts to estimate some parameters that can be used to study the dynamics of the disease. The pandemic data for the period of December 01, 2020 to February 07, 2021 provided by the Nigeria Centre for Disease Control (NCDC) and accessible on the Wolfram Data Repository was use in this research [19, 20]. Here, we present a Susceptible-Exposed-Infectious-Recovered-Deceased (SEIRD) model for analyzing the dynamics of the COVID-19 pandemic in Nigeria. The lack of vaccine has significantly reduced the options for controlling the pandemic in Nigeria. Current control policies are now focused on 'social distancing', 'mask usage' and 'personal hygiene' designed to reduce transmission of the virus. Our proposed model incorporates a control parameter $\mu \in[0,1]$ to indicate the effectiveness of these efforts. Increase in the value of $\mu$ implies improved effectiveness of the control efforts. The objectives of this research include the following: exploration of the impacts of the control policies on the spread of the pandemic, estimation of the infection rate, average latent time, average recovery rate and average mortality rate. The basic reproduction number of the second wave of the disease in Nigeria is also estimated. Predictions on the turning points and possible vanishing time of the virus in Nigeria are made. Recommendations on how to manage the resurgence of the disease are also suggested.

\section{Model and method}

In this section, we present a five-state compartmental model for the analysis of the dynamics of the second wave of the COVID-19 pandemic in Nigeria. It consists of Susceptible $S(t)$, Exposed $E(t)$, Infected $I(t)$, Recovered $R(t)$ and Deceased $D(t)$ compartments denoting at time $t$ the respective number of the susceptible cases, exposed cases, infectious cases, recovered cases and deceased cases. This model shall be referred to as SEIRD model. The description of the interactions between the states is represented by the system of ordinary differential equations given as

$$
\left.\begin{array}{r}
\frac{d S}{d t}=-(1-\mu) \beta S(t) \lambda(t), \\
\frac{d E}{d t}=(1-\mu) \beta S(t) \lambda(t)-\gamma E(t)-\delta_{1} E(t), \\
\frac{d I}{d t}=\gamma E(t)-\delta_{2} I(t)-\sigma I(t), \\
\frac{d R}{d t}=\delta_{1} E(t)+\delta_{2} I(t), \\
\frac{d D}{d t}=\sigma I(t),
\end{array}\right\}
$$

where $\mu \in[0,1]$ is the measure of the overall effectiveness of the current control policies, $\lambda(t)=\frac{I(t)}{N-D(t)}$ is the force of infection. For a communicable disease like the COVID-19, it is more realistic to consider the force of infection as a fraction of the number of infectious persons with respect to the non-deceased population $N$. The constant $N=S+E+I+R+D$ is the total population, $\beta, \gamma^{-1}, \delta_{1}, \delta_{2}$ and $\sigma$ are 
the infection rate, average latent time (days), average recovery rate of exposed persons, average recovery rate of an infected person and average mortality rate among infected persons respectively. Unlike other models discussed in the literature, this work considered the fact that some persons might have very mild symptoms and can move from the exposed category to the recovered category and will never get sick enough to be in the infectious category. This assumption is based on the fact that when a person gets a viral or bacterial infection, a healthy immune system makes antibodies against one or more components of the virus or bacterium. The antibodies help fight the infection, but they might not offer protection against getting reinfected in the future.

\section{Parameter estimation}

Estimation of the model parameters is crucial in understanding the dynamics of the pandemic. In this section, (2.1) is first solved in terms of the model parameters. To obtain initial values needed in the curve fitting, the parameters were manually tuned to get the initial guess. The solution of the model with the

Table 1: Initial values of model parameters.

\begin{tabular}{llllll}
\hline$\mu$ & $\beta$ & $\gamma$ & $\delta_{1}$ & $\delta_{2}$ & $\sigma$ \\
\hline 0.4802248 & 0.6556878 & 0.0445182 & 0.0035958 & 0.0453949 & 0.0002931 \\
\hline
\end{tabular}

initial parameter values is seen in Figure 1.

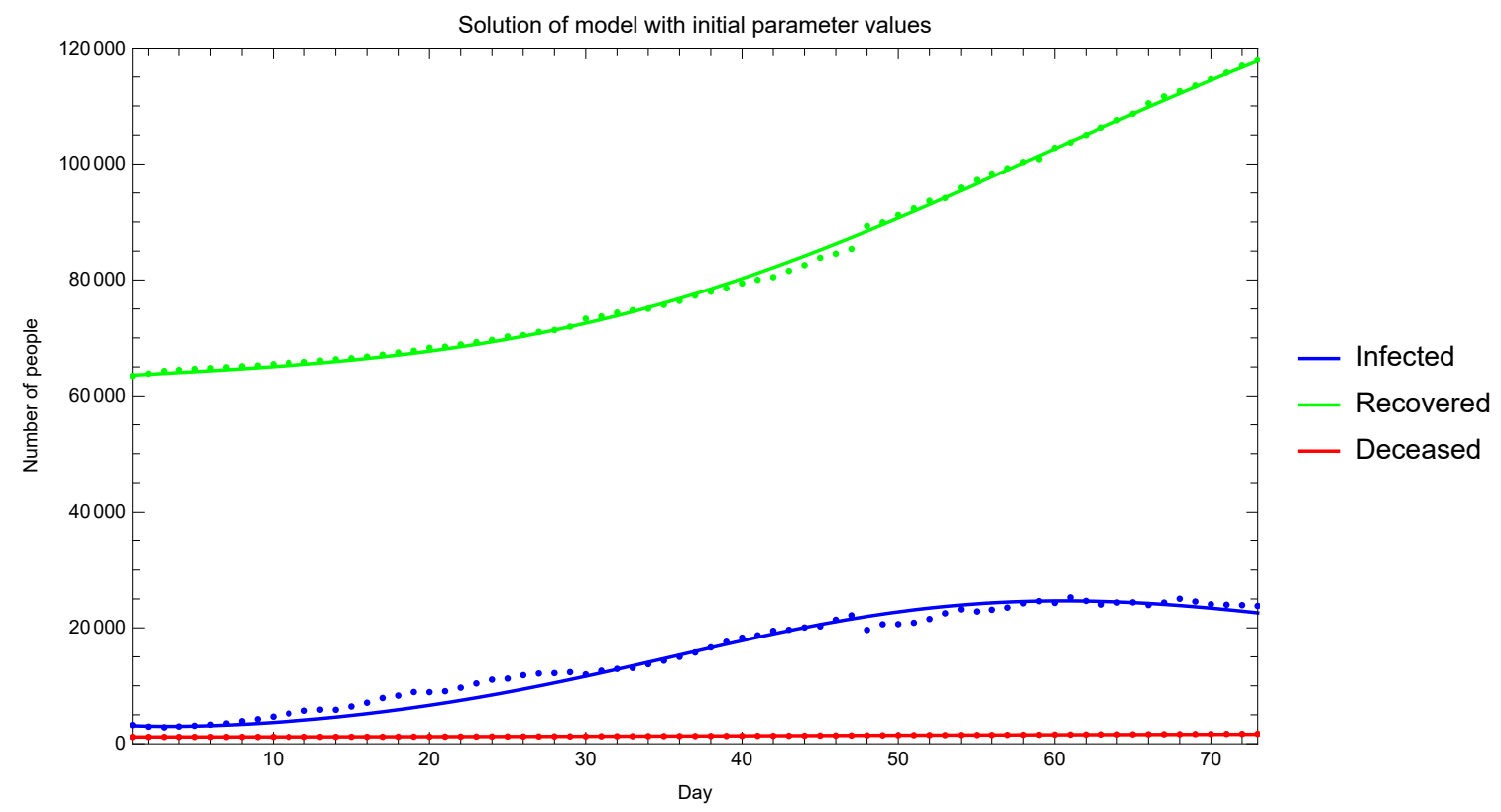

Figure 1: Model solution with initial parameter values.

To obtain the best estimated values of the parameters that appropriately fits the available data, we use the Quasi-Newton algorithm to obtain optimal estimated values of the parameters. The best parameter estimates that fit the data as obtained are presented in Table 2.

Table 2: Estimated values of model parameters.

\begin{tabular}{llllll}
\hline$\mu$ & $\beta$ & $\gamma$ & $\delta_{1}$ & $\delta_{2}$ & $\sigma$ \\
\hline 0.4790797 & 0.6855964 & 0.0422891 & 0.0030572 & 0.0459513 & 0.0002737 \\
\hline
\end{tabular}


The standard error, t-Statistic, p-value and confidence interval of each estimate of the model parameter are given in Table 3.

Table 3: Optimal estimated values of model parameters.

\begin{tabular}{llllll}
\hline Parameter & Estimate & Standard Error & t-Statistic & P-Value & Confidence Interval \\
\hline$\mu$ & 0.4790797 & 0.0021081 & 227.2548 & $3.6424229 \times 10^{-255}$ & $(0.474924,0.483235)$ \\
\hline$\beta$ & 0.6855964 & 0.0079689 & 86.0345 & $7.6802054 \times 10^{-167}$ & $(0.669888,0.701305)$ \\
\hline$\gamma$ & 0.0422891 & 0.0007791 & 54.2775 & $2.5494184 \times 10^{-126}$ & $(0.040753,0.043825)$ \\
\hline$\delta_{1}$ & 0.0030572 & 0.0007459 & 4.0984 & $5.9228000 \times 10^{-005}$ & $(0.001587,0.004528)$ \\
\hline$\delta_{2}$ & 0.0459513 & 0.0012561 & 36.5828 & $1.5042285 \times 10^{-093}$ & $(0.043475,0.048427)$ \\
\hline$\sigma$ & 0.0002737 & 0.0001120 & 2.4441 & $1.5338400 \times 10^{-002}$ & $(0.000053,0.000494)$ \\
\hline
\end{tabular}

\section{Interpretation of model solution}

The dynamics of the second wave of the pandemic in Nigeria is described by the 5-compartmental model (2.1) presented in this work. Optimal estimates of the model parameters show the rates of movements from one compartment to another. The model solution indicates that infected curve is likely to peak in late February, 2021 and the mortality curve is fast approaching flattening around mid February, 2021. It can be seen that effectiveness of the control policies are central in the quick peaking and fastflattening of the curves. The infection rate was estimated as $\beta=0.6855964$ and the overall effectiveness of the control policies was approximately estimated as $47.91 \%$. The average latent time of the disease was estimated as $\gamma^{-1} \approx 23$ days while the average infective time was estimated as $\delta_{2}^{-1} \approx 22$ days. The mortality rate was measured as $\sigma=0.0002737$. The basic reproduction number $\left(R_{0}\right)$ which represents the number of secondary infections in the population caused by one initial primary infection is given by $\mathrm{R}_{0}=\frac{(1-\mu) \beta}{\delta_{2}-\sigma} \approx 8$.

\section{Sensitivity analysis}

In order to determine how well the model fits the data, we present a variety of goodness-of-fit statistics. In this work, the R-Squared statistic is calculated as $99.97254 \%$. The asymptotic parameter correction and covariance matrices are respectively given in Table 4 and Table 5. The fitted curve based on the optimal parameter estimates can be seen in Figure 2.

Table 4: Asymptotic parameter correction matrix.

\begin{tabular}{lllllll}
\hline & $\mu$ & $\beta$ & $\gamma$ & $\delta_{1}$ & $\delta_{2}$ & $\sigma$ \\
\hline$\mu$ & 1. & 0.104884 & 0.0831307 & 0.227435 & -0.224832 & -0.0475515 \\
\hline$\beta$ & 0.104884 & 1. & -0.49581 & 0.0590378 & -0.233606 & -0.140426 \\
\hline$\gamma$ & 0.0831307 & -0.49581 & 1. & -0.782047 & 0.842937 & 0.160654 \\
\hline$\delta_{1}$ & 0.227435 & 0.0590378 & -0.782047 & 1. & -0.953537 & 0.00878173 \\
\hline$\delta_{2}$ & -0.224832 & -0.233606 & 0.842937 & -0.953537 & 1. & 0.00147833 \\
\hline$\sigma$ & -0.0475515 & -0.140426 & 0.160654 & 0.00878173 & 0.00147833 & 1. \\
\hline
\end{tabular}


Table 5: Parameter covariance matrix.

\begin{tabular}{|c|c|c|c|c|c|c|}
\hline & $\mu$ & $\beta$ & $\gamma$ & $\delta_{1}$ & $\delta_{2}$ & $\sigma$ \\
\hline$\mu$ & $4.4442 \times 10^{-06}$ & $1.7620 \times 10^{-06}$ & $1.3654 \times 10^{-07}$ & $3.5765 \times 10^{-07}$ & $-5.9535 \times 10^{-07}$ & $-1.1226 \times 10^{-08}$ \\
\hline$\beta$ & $1.7620 \times 10^{-06}$ & $6.3503 \times 10^{-5}$ & $-3.0784 \times 10^{-06}$ & $3.5094 \times 10^{-07}$ & $-2.3383 \times 10^{-06}$ & $-1.2531 \times 10^{-07}$ \\
\hline$\gamma$ & $1.3654 \times 10^{-07}$ & $-3.0784 \times 10^{-06}$ & $6.0704 \times 10^{-07}$ & $-4.5452 \times 10^{-07}$ & $8.2494 \times 10^{-07}$ & $1.4017 \times 10^{-08}$ \\
\hline$\delta_{1}$ & $3.5765 \times 10^{-07}$ & $3.5094 \times 10^{-07}$ & $-4.5452 \times 10^{-07}$ & $5.5644 \times 10^{-07}$ & $-8.9344 \times 10^{-07}$ & $7.3358 \times 10^{-10}$ \\
\hline$\delta_{2}$ & $-5.9535 \times 10^{-07}$ & $-2.3383 \times 10^{-06}$ & $8.2494 \times 10^{-07}$ & $-8.9344 \times 10^{-07}$ & $1.5778 \times 10^{-06}$ & $2.0795 \times 10^{-10}$ \\
\hline$\sigma$ & $-1.1226 \times 10^{-08}$ & $-1.2531 \times 10^{-07}$ & $1.4017 \times 10^{-08}$ & $7.3358 \times 10^{-10}$ & $2.0795 \times 10^{-10}$ & $1.2540 \times 10^{-08}$ \\
\hline
\end{tabular}

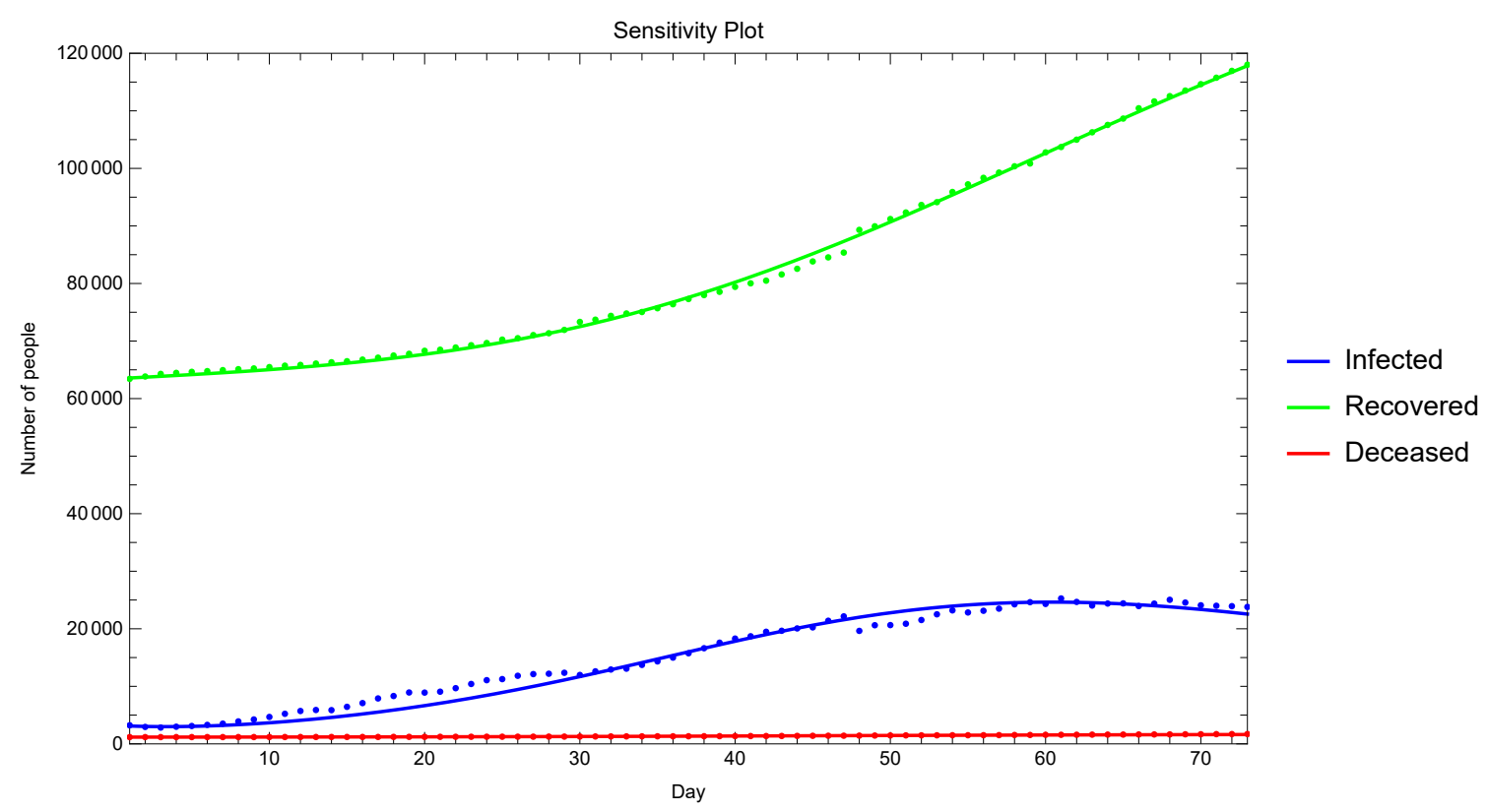

Figure 2: Sensitivity plot with optimal parameter estimetes.

\section{Conclusion and recommendation}

A SEIRD model (2.1) for analysing the dynamics of the second wave of the COVID-19 pandemic in Nigeria has been presented in this work. The model was used to obtain estimates of major parameters: the overall effectiveness of the control policies, infection rate, average latent time, average infective time and the mortality rate influencing the dynamics of the disease. The model fits the deceased compartment well. Also the infected and recovered curves fit the data almost exactly. The R-Squared statistic was calculated as $99.97254 \%$. The infection rate was estimated as $\beta=0.6855964$ and the overall effectiveness of the control policies was approximately estimated as $47.91 \%$. The average latent time of the disease was estimated as $\gamma^{-1} \approx 23$ days while the average infective time was estimated as $\delta_{2}^{-1} \approx 22$ days. The mortality rate was measured as $\sigma=0.0002737$. The basic reproduction number which was estimated as $\mathrm{R}_{0} \approx 8>1$ clearly shows that the disease is already spreading. It is clear that the control policies are not properly adhered to. We recommend a strict enforcement of the control policies in order to help manage the resurgence and ultimately flatten the curves of the second wave of the pandemic in Nigeria.

\section{References}

[1] T. G. Bryan, A. Kleczkowski, C. A. Gilligan, B. M. Bolker, Spatial heterogeneity, non-linear dynamics and chaos in infectious diseases, Stat. Methods Medical Res., 4 (1995), 160-183. 1 
[2] E. Dong, H. Du, L. Gardner, An interactive web-based dashboard to track COVID-19 in real time, Lancet Infect Dis., 20 (2020), 533-534. 1

[3] R. Engbert, M. M. Rabe, R. Kliegl, S. Reich, Sequential data assimilation of the stochastic SEIR epidemic model for regional COVID-19 dynamics, Bull. Math. Biol., 83 (2021), 16 pages. 1

[4] J. Espigule-Pons, Mapping Novel Coronavirus COVID-19 Outbreak, Wolfram Community, (2020). 1

[5] T. Götz, First attempts to model the dynamics of the coronavirus outbreak, arXiv, 2020 (2020), 13 pages. 1

[6] L. E. Gralinski, V. D. Menachery, Return of the Coronavirus: 2019-nCoV, Viruses, 12 (2020), 8 pages.

[7] E. Grigorieva, E. Khailov, A. Korobeinikov, Optimal control for an SEIR epidemic model with nonlinear incidence rate, Stud. Appl. Math., 141 (2018), 353-398. 1

[8] A. Hornstein, Social Distancing, Quarantine, Contact Tracing, and Testing: Implications of an Augmented SEIR-Mode, Technical Report, Federal Reserve Bank of Richmond, (2020). 1

[9] J. Jia, J. Ding, S. Liu, G. Liao, J. Li, B. Duan, G. Wang, R. Zhang, Modeling the Control of COVID-19: Impact of Policy Interventions and Meteorological Factors, arXiv, 2020 (2020), 21 pages. 1

[10] W. O. Kermack, A. G. McKendrick, A contribution to the mathematical theory of epidemics, Proc. R. Soc. Lond. A, 115 (1927), 700-721. 1

[11] M. A. Khan, Y. Khan, S. Islam, Complex dynamics of an SEIR epidemic model with saturated incidence rate and treatment, Phys. A, 493 (2018), 210-227. 1

[12] F. Y. Liew, J. S. Dhaliwal, Distinctive cellular immunity in genetically susceptible BALB/c mice recovered from Leishmania major infection or after subcutaneous immunization with killed parasites, J. Immuno., 138 (1987), 4450-4456.

[13] Q. Liu, D. Q. Jiang, N. Z. Shi, T. Hayat, B. Ahmad, Stationary distribution and extinction of a stochastic SEIR epidemic model with standard incidence, Phys. A, 476 (2017), 58-69. 1

[14] Q. Liu, D. Q. Jiang, N. Z. Shi, T. Hayat, A. Alsaedi, Asymptotic behavior of a stochastic delayed SEIR epidemic model with nonlinear incidence, Phys. A, 462 (2016), 870-882. 1

[15] L. Peng, W. Yang, D. Zhang, C. Zhugeand, L. Hong, Epidemic analysis of COVID-19 in China by dynamical modeling, arXiv, 2020 (2020) 11 pages. 1

[16] N. Sharma, A. K. Gupta, Impact of time delay on the dynamics of SEIR epidemic model using cellular automata, Phys. A, 471 (2017), 114-125. 1

[17] A. Takaki, M. Wiese, G. Maertens, E. Depla, U. Seifert, A. Liebetrau, B. Rehermann, Cellular immune responses persist and humoral responses decrease two decades after recovery from a single-source outbreak of hepatitis $C$, Nature Medicine, 6 (2000), 578-582.

[18] X. W. Wang, H. J. Peng, B. Y. Shi, D. H. Jiang, S. Zhang, B. S. Chen, Optimal vaccination strategy of a constrained time-varying SEIR epidemic model, Commun. Nonlinear Sci. Numer. Simul., 67 (2018), 37-48. 1

[19] A. S. Wusu, O. A. Olabanjo, SEIRD Model for Analyzing Coronavirus (COVID-19) Pandemic, Wolfram Demonstrations Project, (2020). 1

[20] A. S. Wusu, O. A. Olabanjo, SIRD Model for Analyzing Coronavirus (COVID-19) Pandemic, Wolfram Demonstrations Project, (2021). 1

[21] Y. Xue, G. Zhang, H. Pang, Y. Zhou, R. Wang, Forecasting and Analysis of Time Variation of Parameters of COVID-19 Infection in China Using An Improved SEIR Model, Research Square, 2020 (2020), 6 pages. 1

[22] Y. Zhou, Z. Chen, X. Wu, Z. Tian, L. Cheng, L. Ye, The Outbreak Evaluation of COVID-19 in Wuhan District of China, arXiv, 2020 (2020), 11 pages. 1 